\title{
Research of Electromagnetic Wave Energy-Harvesting Technology on Free Space
}

\author{
College of Information Engineering, Jimei University \\ Xiamen, Fujian, 361021, China. \\ *Corresponding Author: 201761000018@jmu.edu.cn
}

Zhong-Hua Ma, Chih-Cheng Chen*, Chang-Bing Huang, Guang-Song Yang

\begin{abstract}
In order to provide long-term power supply for low-power consumption and many wireless sensor nodes, it is proposed to replace the battery and the power supply line by harvesting space radio wave energy to make up for the trouble of replacing the battery and reduce environmental pollution. Dickson rectifier voltage multiplier circuit is constructed by using schottky diode with low conduction voltage when a radio frequency of $400 \mathrm{MHz}$ and a voltage amplitude of $5 \mathrm{mV}$ is input, the rectified output DC voltage reaches $20 \mathrm{uV}$. This scheme provides a reference for wireless power supply of low power class circuits.
\end{abstract}

Key words: Rectification, energy-harvesting, matching circuit, Schottky diodes

\section{Introduction}

With the depletion of non-renewable fossil resources such as oil, coal and natural gas, the development and research of new energy has been highly valued by governments and scholars. During the use of fossil energy, a large amount of carbon dioxide and sulfur dioxide, dust and other polluting gases and pollutants will be generated, causing air pollution and greenhouse effect, leading to climate warming.

In order to solve the energy crisis, scientists from all over the world are looking for sustainable clean energy. In 1968, American scientist Peter Glaser proposed the solar satellite program, which was to set up a satellite solar power station, use a huge solar array to convert solar energy into electricity in space. Bringing powerful solar energy back to Earth through wireless transmission $\left.{ }^{[1,} 2\right]$. This microwave power transmission (MPT) technology reduces the weight and volume of energy users. The transmission loss is only atmospheric loss, rain attenuation and obstruction loss. Among them, the solar energy plan in the concept of wireless energy transmission has attracted the attention of governments and experts in various countries, and has launched research in related fields, which has greatly promoted the development of microwave transmission technology.

The application field of microwave transmission has also gradually expanded to many other fields, such as ground power supply system ${ }^{[3]}$, wireless sensors ${ }^{[4]}$, wearable devices ${ }^{[5]}$ and so on. At present, in the homes of residents, offices and other occasions, there are a large number of power supply lines, which not only occupy space, but also cause potential safety hazards, and also cause waste of resources. There are also officers and men stationed in the islands, working at the radar station on the mountain, etc., because it is very inconvenient to erect wires. Their working and living electricity mainly depends on diesel generators, and fuel and other consumables are often handled by hand. Therefore, it is feasible to use microwave power to supply energy to these areas.

Many wireless sensor networks currently have many network nodes and require real-time detection and data processing. The general nodes are all powered by batteries. The available power is very limited, and it is very difficult to replace the batteries for thousands of nodes. Battery costs are high and replacement is difficult, and the environment will be seriously polluted, and the solar cells are obviously too bulky. If the receiving device in microwave power transmission is used to collect the energy of the space radio waves, this inexhaustible energy provides a practical method for powering the micro system, reducing the recycling and landfill of used batteries, and prevents the environmental pollution caused by the battery's chemical materials. RF power capture is small, no more than a few milliwatts, but this energy harvesting technology provides a promising future for low-power consumer electronics, wearable devices, implantable medical devices, and wireless sensor networks.

Although wireless transmission technology is in full swing at home and abroad, the capturing of space-ready radio wave energy is still in the development stage. At present, there is no work in this area in China, and there are few reports on this in foreign countries. British scholar Pinuela realized the energy capturing of DTV, GSM900 and GSM1800, 3G RF waves in urban and suburban environments, and designed four frequency band dipole antennas respectively, the energy collection efficiency reached $40 \%{ }^{[6]}$. Literature [7] reported that Japanese scholars used rectennas to capture RF energy from base stations of mobile communications to power LCD thermometers. Korean scholar Jeong has improved the circuit based on CMOS voltage multiplier, so that the output de power of rectifier antenna increases by 160 percent compared with that of traditional rectifier antenna at $0 \mathrm{dBm}$ power input ${ }^{[8]}$.Two energy capture systems are presented in Reference [9], which use RFID readers and TV antennas to capture energy and obtain $60 \mathrm{uW}$ of power from $4.1 \mathrm{~km}$ away. Reference [10] reported capturing a $470-570 \mathrm{MHz}$ RF power using a rectenna antenna of a dipole antenna printed on paper.

Numerous radio waves in free space are inexhaustible RF energy sources. The use of rectennas to capture the radio wave energy of space can just make up for the shortcomings of traditional MPT technology. Captured space radio frequency (RF) energy can be used to power a wide range of low-power devices such as Microsystems and sensor networks. The most critical part of wireless energy harvesting is the rectenna, 
which consists of a receiving antenna, a matching circuit, a rectifier diode, and a DC load.

\section{RF energy harvesting system}

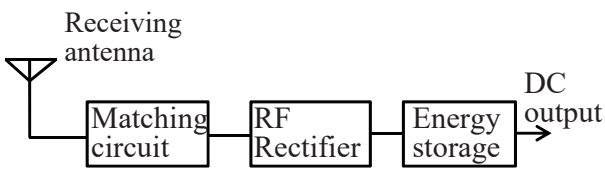

Fig.1 the system of the energy harvesting

The emerge harvesting system is shown in Fig.1. It mainly comprises a receiving antenna, a radio frequency signal rectifying circuit, a matching circuit and an energy storage circuit. The receiving antenna is generally a planar antenna or an omnidirectional antenna. It works for multi-frequency operation or ultra-wideband, and mainly collects various radio frequency signals from base stations of mobile communication, GPS, DTV and various radio stations. The matching circuit performs impedance matching between the receiving antenna and the RF rectifying circuit to improve the efficiency of RF energy collection and the output voltage. The rectifier circuit has the functions of rectification and boosting, converts the RF signal into DC, and stores it in the energy storage circuit for DC output.

Since the energy of various radio wave signals in space is very small, the voltage is generally smaller than the conduction voltage of the ordinary rectifier diode, and the ordinary rectifier diode cannot complete the rectification function of the space electromagnetic wave. The Schottky diode is a metal-semiconductor structure formed by metal-to-semiconductor contact, characterized by short reverse recovery time, very fast switching transition, very low turn-on voltage, low junction capacitance and low equivalent series resistance. It can be used as a rectifier diode and a small signal detection diode in microwave circuits. It can also be used as a rectifier diode in radio wave collection, which is the core of the rectenna.

\section{Design of the rectification circuit}

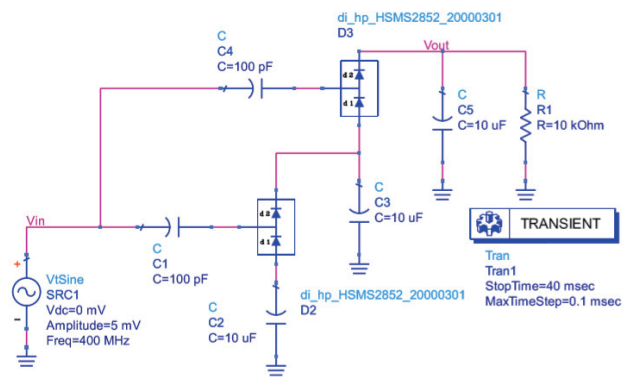

Fig. 2 the rectifier circuit

The Schottky HSMS-2852 diode is used as the rectifier tube, and the structure of the Dickson voltage multiplier is shown in Figure 2.

The radio signal in free space is very weak. To enable the rectifier diode to start, the Schottky diode with low turn-on voltage must be used. Such a weak signal, the output DC after RF-DC conversion is also small. To increase the output voltage, a multi-stage diode rectification boost circuit is required. Increasing the number of voltage multipliers can provide a higher voltage at the load, but after the number of stages of the diode rectification boost circuit reaches a certain number, the voltage gain decreases as the voltage multiplication series increases due to the parasitic effect of the capacitance. The DC voltage is no longer raised.

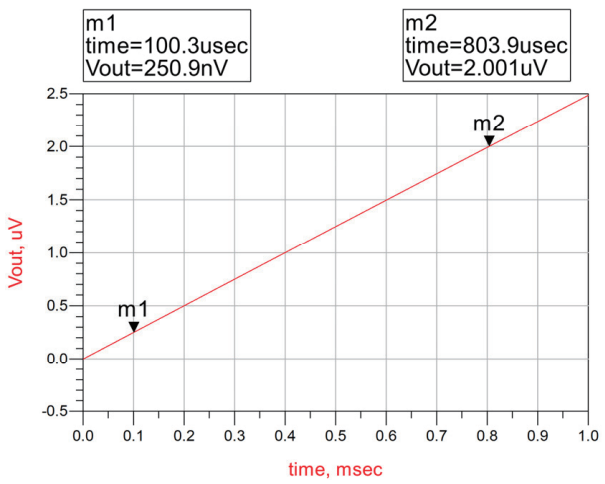

Fig. 3 The direct current output from the second-order rectifier circuit, When the amplitude of the collected electromagnetic wave is $5 \mathrm{mV}$.

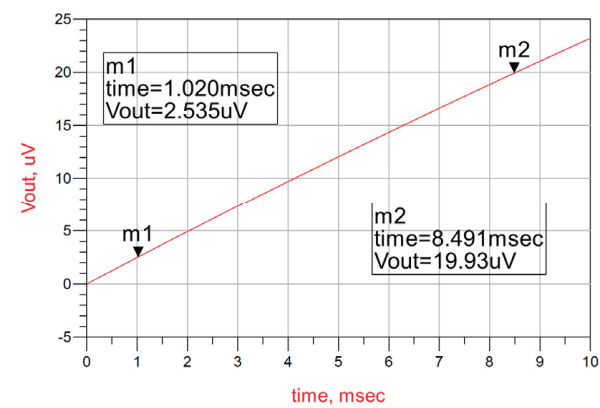

Fig. 4 The direct current output from the nine-stage rectifier circuit,when the amplitude of the collected electromagnetic wave is $5 \mathrm{mV}$.

Figure 3 is a DC voltage output from the second-order Dickson rectifier circuit shown in Figure 2. When the frequency of the electromagnetic wave collected by the receiving antenna is $400 \mathrm{MHz}$ and the voltage amplitude is 5 $\mathrm{mV}$, after the rectification boost circuit, the DC voltage of the output reaches $250 \mathrm{nV}$ at about $100 \mathrm{us}$; after about $800 \mathrm{us}$, the output DC voltage reaches $2 \mathrm{uV}$. Such DC voltages are not sufficient to enable low-power circuits to start, and to supply power to low-power devices and circuits, a larger DC voltage must be provided. Therefore, the second-order Dickson rectifier circuit is increased to the ninth order, and the simulated output DC voltage curve is shown in Fig. 4. After $8.5 \mathrm{~ms}$ of radio wave energy collection, the output DC voltage has reached $20 \mathrm{uV}$, indicating that the output voltage will increase as the order of the rectified voltage multiplier increases. 


\section{Conclusion}

Space radio waves are an inexhaustible off-the-shelf RF energy source that can be converted into DC energy through a rectenna to supply low-power (sensor nodes) and micro-system devices. Power supply systems for low-power intelligent electronic devices such as wearables and micro-weapons are widely used; wireless sensor networks and the Internet of Things, etc.; instead of batteries, a series of power sources that cause serious pollution and cost to the environment. In the future, not only small power appliances, common household appliances, medical equipment, power tools, office appliances and kitchen appliances can be wirelessly powered. In the future, the development trend of all power-consuming equipment must be wireless.

\section{Acknowledgments}

This work was supports by the Xiamen Industry-University-Research Collaboration Project (3502Z20163008) and the Key Project of the Education Department of Fujian Province (JA15257)

\section{References}

[1]. P. E. Glaser. Power from the sun: its future [J]. Science, 1968, 162(3856): 857-861.

[2]. C. T. Rodenbeck and K. Chang. A limitation on the small-scale demonstration of retrodirective microwave power transmission from the solar power satellite [J]. Antennas \& Propagation Magazine IEEE, 2005, 47(4): 67-72.

[3]. A. Celeste, P. Jeanty and G. Pignolet. Case study in Reunion Island [J]. Acta Astronautica, 2004, 54(4): 253-258.

[4]. D. Porcarelli, D. Spenza, D. Brunelli and A. Cammarano. Adaptive Rectifier Driven by Power Intake Predictors for Wind Energy Harvesting Sensor Networks [J]. IEEE Journal of Emerging \& Selected Topics in Power Electronics, 2015, 3(2): 471-482.

[5]. V. Mihajlovic, B. Grundlehner, R. Vullers and J. Penders. Wearable, wireless EEG solutions in daily life applications: what are we missing? [J]. IEEE Journal of Biomedical and Health Informatics, 2015, 19(1): 6-21.

[6]. M. Piñuela, P. D. Mitcheson and S. Lucyszyn. Ambient RF Energy Harvesting in Urban and Semi-Urban Environments [J]. IEEE Transactions on Microwave Theory \& Techniques, 2013, 61(7): 2715-2726.

[7]. S. Kitazawa, H. Ban and K. Kobayashi. Energy harvesting from ambient RF sources [C]. In Microwave Workshop Series on Innovative Wireless Power Transmission: Technologies, Systems, and Applications, 2012: 39-42.

[8]. H. Jabbar, Y. S. Song and T. T. Jeong. RF energy harvesting system and circuits for charging of mobile devices [J]. IEEE Transactions on Consumer Electronics, 2010, 56(1): 247-253.

[9]. A. Sample and J. R. Smith. Experimental results with two wireless power transfer systems [C]. In 2009 IEEE Radio and Wireless Symposium, 2009: 16 - 18.

[10]. R. Vyas, V. Lakafosis, M. Tentzeris, H. Nishimoto and Y. Kawahara. A battery-less, wireless mote for scavenging wireless power at UHF (470-570 MHz) frequencies [C]. In 2011 IEEE International Symposium on Antennas and Propagation (APSURSI), 2011: 1069-1072. 
Recent Developments on Information and

Communication Technology (ICT) Engineering- Meen, Yang \& Zhao ISBN: 978-981-14-2136-5 